\title{
DESCRIPTION ET CARACTERES BIOLOGIQUES DE DEUX NOUVEAUX RHABDIAS MALGACHES
}

\section{Par Alain-G. CHABAUd, Edouard-R. BRYGOO et Annie-d. PETTER (*)}

Deux Rhabdias malgaches ont été étudiés. L'un, assez fréquent chez les Grenouilles, a un cycle hétérogonique assez banal et ne sera décrit que brièvement ; l'autre, qui n'a été rencontré qu'une fois, chez un Caméléon, a des particularités biologiques remarquables. Nous proposons les noms de Rhabdias madagascariensis n. sp. pour le parasite de Rana, et de Rhabdias gemellipara n. sp. pour le parasite de Caméléon.

\section{Rhabdias madagascariensis n. sp.}

\section{$1^{\circ}$ Garactères biologiques.}

Une Rana (Ptychadena) mascareniensis (D. et B.), 40 G, capturée dans les jardins de l'Institut Pasteur de Tananarive le 6 avril 1961, contient six Rhabdias (femelles mûres) dans les poumons. Une coproculture est faite en diluant, dans une boîte de pétri, le contenu rectal assez pauvre en larves rhabditoïdes, avec un peu de charbon. Conservée à une température de $20^{\circ}$, cette coproculture reste très pauvre. Un petit nombre d'adultes libres sont observés très précocement (mâles au bout de $\mathbf{2 4}$ heures, femelles mûres au bout de 44 heures). Il semble ne pas y avoir eu de cycle direct, car c'est seulement le $9^{\circ}$ jour qu'une larve filariforme est observée.

\section{$2^{\circ}$ Femelle parasite.}

Les spécimens types proviennent des poumons d'une Rana (Ptychadena) mascareniensis (D. et B.) 188 E, trouvée le 6 octobre 1957 dans les jardins de l'Institut Pasteur de Tananarive. Le corps est long de $3,55 \mathrm{~mm}$. et atteint dans la région vulvaire une largeur maximum de $150 \mu$. Bouche arrondie, sans lèvres. Papilles et amphi-

(1) Travail effectué grâce à une subvention pour frais de voyages accordée par le Centre National de la Recherche Scientifique. 
des très peu saillantes et difficiles à voir. Un très petit anneau chitinoïde se trouve en avant de l'œsophage, mais il n'est perceptible qu'en vue apicale. L'œsophage, long de $270 \mu$, est constitué de deux portions bien distinctes : l'antérieure, longue de $70 \mu$, forme un tube cylindrique étroit ; la postérieure, longue de $200 \mu$, est nettement plus large et se dilate progressivement en massue en arrière. L'anneau nerveux, à $130 \mu$ de l'apex, est nettement postérieur au niveau

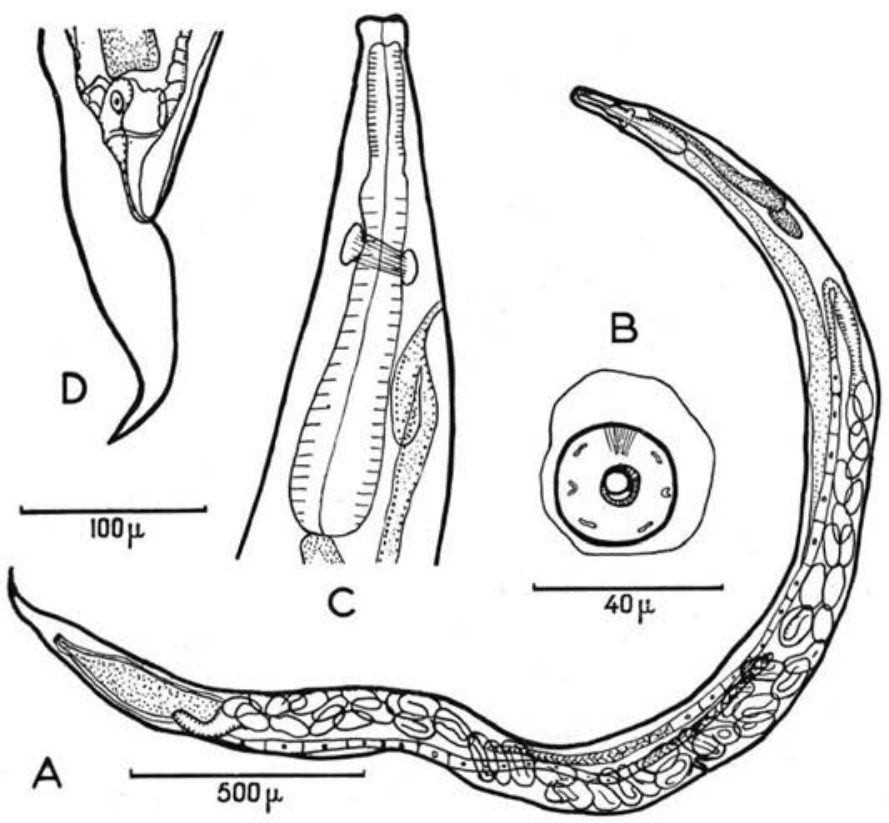

Fig. 1. - Rhabdias madagascariensis, Femelle parasite. A : Corps entier, vue latérale. - B : Tête, vue apicale. C : Extrémité antérieure, vue latérale. - D : Extrémité postérieure, vue latérale.

de jonction des deux portions œesophagiennes. Le pore excréteur, situé à $155 \mu$ de l'apex, est relié à un sinus long de $60 \mu$, d'où partent deux énormes glandes subventrales longues de 350 à $400 \mu$.

La vulve, non saillante, est située à $1,85 \mathrm{~mm}$. de l'extrémité antérieure. Les utérus contiennent de très nombreux œufs d'environ $110 \mu \times 50 \mu$, qui se transforment en larves dans les portions proches de la vulve. La disposition des oviductes et des ovaires est proche de celle qui est figurée par Goodey (1924a) pour $R h$. fuscovenosa. La queue, longue de $125 \mu$, est conique très aiguë. 


\section{$3^{\circ}$ Femelle libre.}

La coproculture étant très pauvre, nous n'avons pas pu étudier les différents stades larvaires et devons nous limiter aux adultes libres.

Une jeune femelle prélevée à la $44^{\circ}$ heure a pour principales dimensions : longueur $610 \mu$, largeur $30 \mu$; œsophage long de $148 \mu$ avec corpus, isthme et bulbe longs respectivement de 88,30 et $30 \mu$. Anneau nerveux et pore excréteur respectivement à 108 et $123 \mu$ de l'apex. Vulve à $350 \mu$ de l'extrémité antérieure. Queue longue de $60 \mu$. Les utérus contiennent 12 œufs non segmentés, dont un de grande taille.

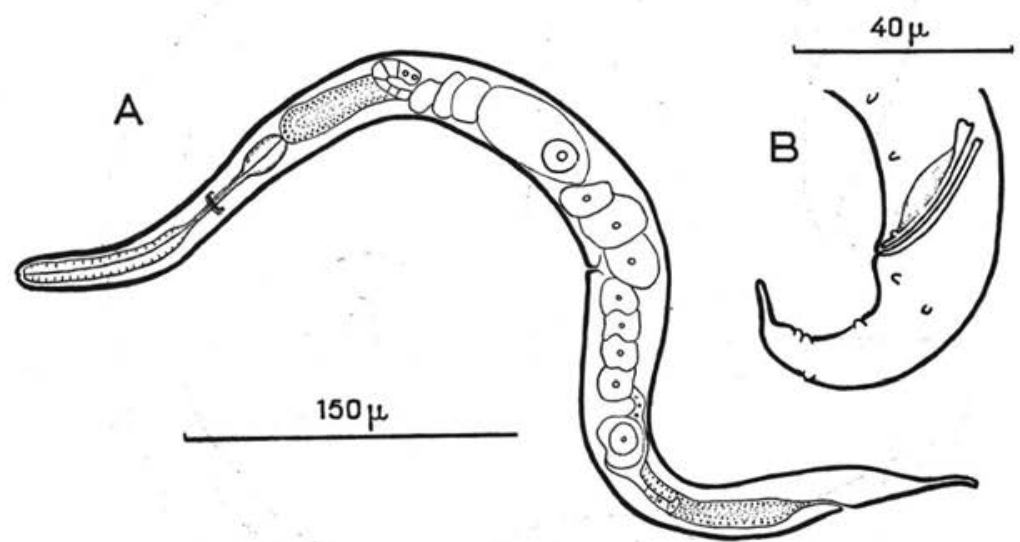

Fig. 2. - Rhabdias madagascariensis. Adultes libres. A: Fentelle ( $44^{\circ}$ heure), vue latérale. - B : Mâle $\left(24^{\circ}\right.$ heure), extrémité postérieure, vue latérale.

\section{$4^{\circ}$ Mâle libre.}

Un mâle prélevé à la $24^{\circ}$ heure est long de $720 \mu$ et large de $23 \mu$. Esophage long de $160 \mu$ avec corpus, isthme et bulbe longs respectivement de $90 \mu, 38 \mu$ et $32 \mu$. Queue longue de $48 \mu$, sans ailes caudales ; son extrémité, brusquement rétrécie, forme une pointe longue de $8 \mu$. Les spicules sont subégaux $(28 \mu)$, mais dissemblables, car l'un porte une aile assez large, alors que le second, un peu plus fin, en est dépourvu. La disposition des papilles cloacales est indiquée sur la figure $2 \mathrm{~B}$. Si l'on admet la nomenclature que nous avons récemment proposée (1961), c'est une disposition très primitive, avec une paire apparemment absente, la paire 4 un peu déportée en arrière de la 5, et la 8 en position non modifiée. 


\section{Rhabdias gemellipara n. sp.}

\section{$1^{\circ}$ Garactères biologiques.}

Un Chamaeleo parsonii Cuvier 1825, 11 G, capturé à Périnet le $1^{\text {er }}$ avril 1961, est parasité par un Rhabdias femelle dans le poumon et a des larves rhabditoïdes dans les matières fécales. Une coproculture préparée comme précédemment est également conservée à une timpérature de $20^{\circ}$. L'évolution est très rapide ; des larves du 4 " stade sont déjà formées au bout de 24 heures et des femelles ovifères sont visibles au bout de 48 heures. A ce stade, chacun des utérus contient une grosse ébauche déjà vermiıorme en position distale, près de la vulve, puis un œui de taille moyenne, au stade 4 ou 8 blastomères, puis 3 à 6 ovules petits et non segmentés.

Le troisième jour, l'organisme maternel est complètement détruit, mais la cuticule est intacte. A l'intérieur'se trouvent deux larves à œsophage rhabditoïde qui s'agitent dans un liquide opaque contenant de nombreuses granulations fines. Le quatrième jour, au contraire, le liquide contenu dans la cuticule maternelle est entièrement digéré et devient tout à fait transparent. Une mue (apparemment la première mue) a lieu avant le cinquième jour. Les deux larves emprisonnées dans la cuticule maternelle prennent alors un œsophage filaroïde et restent encloses dans la cuticule précédente où elles deviennent mûres (apparemment fin du $2^{\circ}$ stade). Ces larves, qui jusqu'alors étaient très actives, deviennent immobiles. A quelques exceptions près, elles ne s'échappent ni de l'étui individuel formé par la cuticule du $1^{\text {er }}$ stade, ni de l'enveloppe formée par la cuticule maternelle où elles restent groupées deux par deux. Nous n'avons jamais vu de femelles contenant une seule larve, ou de femelles contenant trois larves. Par contre, alors que, depuis le 3 ou $4^{\circ}$ jour, toutes les femelles semblaient réduites à un étui cuticulaire, nous avons observé le $12^{\circ}$ jour une femelle encore vivante et mobile, contenant des œufs morts. L'avortement permet donc une survie relativement prolongée des femelles. Jusqu'au $15^{\circ}$ jour, ces larves vivantes ont été observées régulièrement dans la coproculture. Elles conservent une immobilité stricte pendant le prélèvement, mais, après un éclairage violent d'environ une minute sous la loupe binoculaire, elles reprennent une très forte activité à l'intérieur de la cuticule maternelle.

De très nombreux Chamaeleo lateralis Gray 1831, capturés dans les jardins de l'Institut Pasteur de Tananarive, ont été examinés et ont toujours été négatifs. Trois spécimens de la même provenance sont infestés par voie buccale, le $12^{\circ}$ jour, avec les larves de la 
coproculture (1). L'un ayant reçu environ 15 larves est négatif à l'autopsie, quatre jours après. Le deuxième, infesté par une dose analogue, est également négatif au bout d'un mois. Par contre, le troisième, qui a été infesté par environ 30 larves, présente, un mois après, cinq Rhabdias dans les poumons. Ces parasites sont moins grands que le matériel original provenant de $C$. parsonii $(4,65 \mathrm{~mm}$. au lieu de $8,41 \mathrm{~mm}$.), mais ils présentent les mêmes caractères et ont dans les utérus un nombre d'œufs comparable.

\section{$2^{\circ}$ Femelle parasite.}

La femelle holotype, prise dans le poumon de Chamaeleo parsonii, à Périnet, le $1^{\text {er }}$ avril 1961 , est longue de $8,41 \mathrm{~mm}$. Sa largeur maximum est de $205 \mu$. Le corps est recouvert d'une cuticule lâche et irrégulière qui ressemble au «teguminal sheath 》 des Métastrongylides. Bouche arrondie, sans lèvres. Il existe, en avant de l'œsophage, une véritable capsule buccale, bien chitinoïde, mais très petite (diamètre externe $17 \mu$, hauteur $8 \mu$ ).

L'œsophage, long de $630 \mu$, comprend deux portions distinctes par leur structure, mais de diamètre peu différent. La partie antérieure, longue de $190 \mu$, est faiblement dilatée en arrière ; la partie postérieure, longue de $440 \mu$, est presque cylindrique, puis forme un léger bulbe terminal. L'anneau nerveux entoure l'extrémité antérieure de l'œsophage postérieur, à $210 \mu$ de l'apex. Le système excréteur, qui était remarquablement grand et bien visible sur l'espèce précédente, est ici très peu apparent; la position du pore excréteur n'a pu être précisée.

La vulve est située à $4,28 \mathrm{~mm}$. de l'extrémité antérieure. L'anatomie génitale est comparable à celle de l'espèce précédente. Les œufs sont assez peu nombreux, ils mesurent environ $100 \mu \times 50 \mu$ et sont à un stade d'évolution peu avancé. La queue, longue de $300 \mu$, se termine en pointe très fine.

Les spécimens obtenus expérimentalement chez Chamaeleo lateralis, ont des dimensions très différentes : longueur $4,65 \mathrm{~mm}$., largeur $150 \mu$. CEsophage $515 \mu$. Anneau nerveux à $185 \mu$ de l'apex. Vulve à $2,55 \mathrm{~mm}$. de l'extrémité antérieure. Queue longue de $220 \mu$.

\section{$3^{\circ}$ Larve rhabditoïde.}

Une larve prélevée dans les matières fécales de $C$. parsonii, avant la mise en coproculture, est longue de $470 \mu$ et large de $25 \mu$. L'œso-

(1) La contamination directe par voie buccale s'effectue vraisemblablement sans difficulté, car les Caméléons en captivité boivent avidement les gouttes d'eau déposées sur les parois de la cage, et il est probable que dans la nature ils ingèrent les gouttes de rosée qui se forment à proximịté du gíte. 
phage, long de $120 \mu$, comprend un pharynx, un corpus, un isthme et un bulbe longs respectivement de $8 \mu, 68 \mu, 22 \mu$ et $22 \mu$. L'anneau nerveux est à $88 \mu$ de l'apex. La queue est longue de $57 \mu$. L'ébauche génitale, longue de $30 \mu$, a sa partie moyenne située à $240 \mu$ de l'extrémité antérieure. La cuticule est marquée de stries transversales simples.

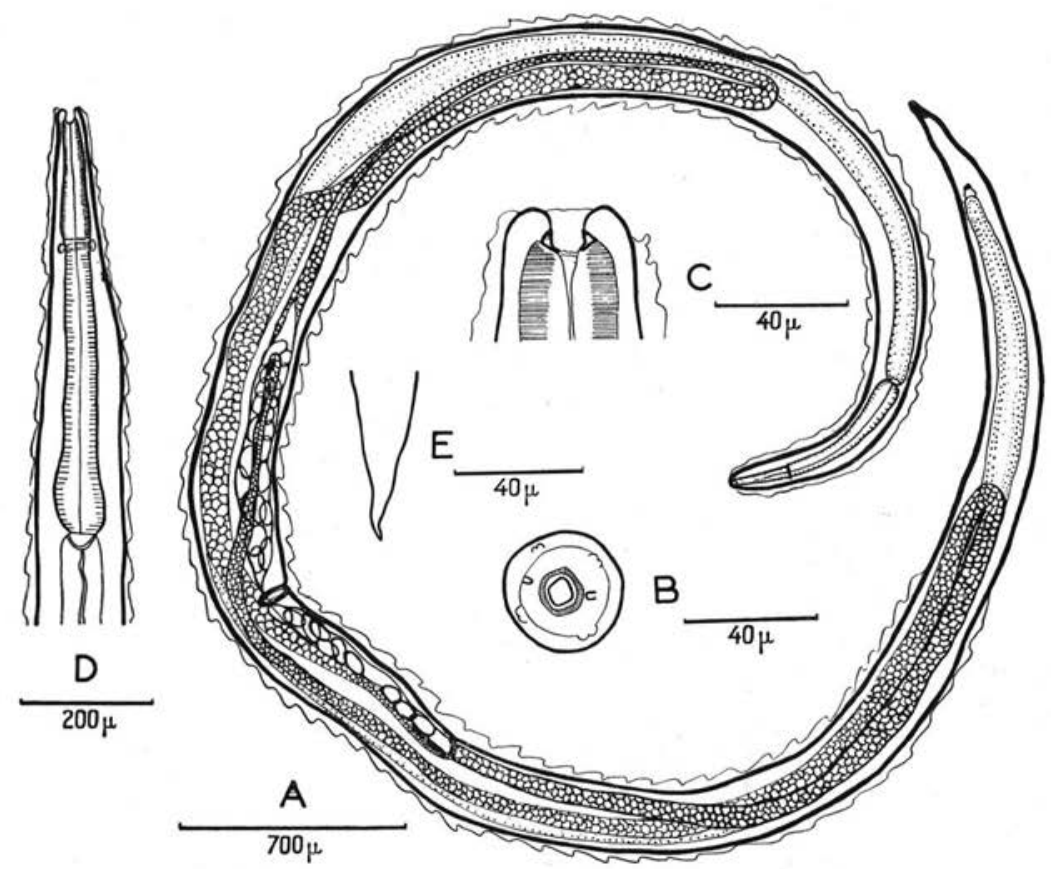

Fig. 3. - Rhabdias gemellipara. Femelle parasite. A : Corps entier, vue latérale. - B : Tête, vue apicale (petit spécimen de l'infestation expérimeñtale). - C : Extrémité céphalique, vue latérale. - D : Extrémité antérieure, vue latérale. - E : Pointe caudale.

\section{$4^{\circ}$ Femelle libre.}

Une femelle prélevée dans la coproculture à la $48^{\circ}$ heure est longue de $1,35 \mathrm{~mm}$. et large de $75 \mu$. L'œsophage, long de $208 \mu$, comprend un pharynx, un corpus, un isthme et un bulbe longs respectivement de $10 \mu, 112 \mu, 48 \mu$ et $38 \mu$. Anneau nerveux et pore excréteur respectivement à 160 et $198 \mu$ de l'apex. Vulve à $730 \mu$ de l'extrémité antérieure. Le grand œuf contenu dans chaque utérus mesure $100 \times 48 \mu$. La queue, très aiguë, est longue de $130 \mu$. 


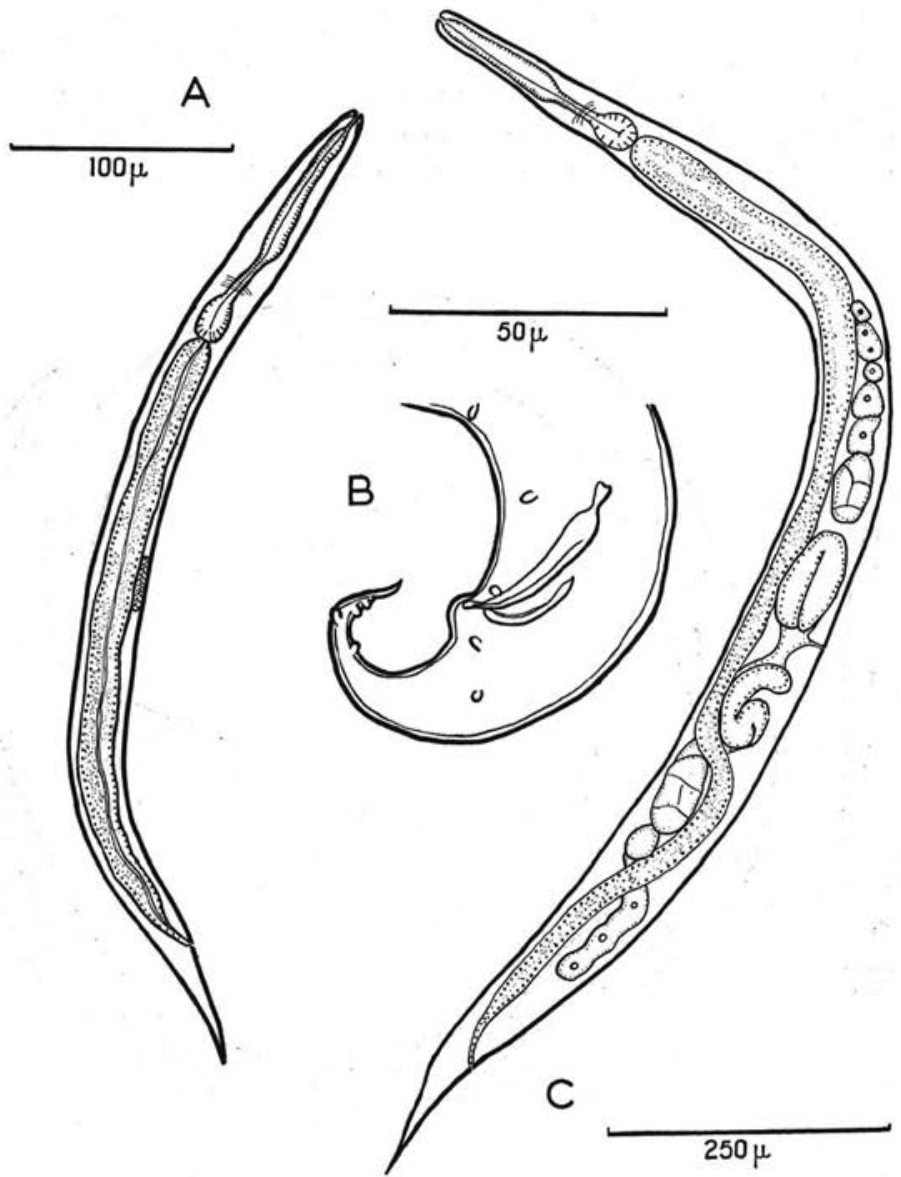

FIG. 4. - Rhabdias gemellipara. Formes libres. A : Larve prélevée dans les matières fécales. - B : Extrémité postérieure du mâle (48 heure), vue latérale. - C : Femelle (48 heure).

\section{$5^{\circ}$ Mâle libre.}

Les mensurations du mâle n'ayant, par erreur, pas été faites, nous donnons celles d'une larve mâle du $4^{\circ}$ stade, prise dans la coproculture de 24 heures, au moment où la mue définitive s'effectue. Longueur : $810 \mu$; largeur : $32 \mu$; œsophage long de $165 \mu$ avec pharynx de $10 \mu$, corpus de $108 \mu$, isthme de $20 \mu$ et bulbe de $27 \mu$. Queue longue de $62 \mu$. Spicules de $32 \mu$ et $17 \mu$. Sur un mâle adulte, à la $48^{\circ}$ heure, les dimensions de la queue et des spicules ne sont pas modifiées. Le cloaque forme une bosse saillante. Il y a 10 paires 
de papilles, avec les paires 4 et 8 dorsales, la 4 étant un peu en arrière de la 5 . Les spicules sont beaucoup plus inégaux que chez l'espèce précédente.

\section{$6^{\circ}$ Larve rhabditoïde hétérogenique.}

Les larves, libérées artificiellement de la cuticule maternelle le

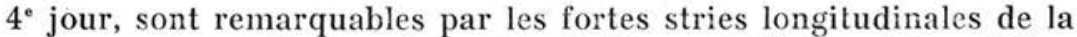
cuticule. Il en existe environ 16 qui débutent dès la région cépha-
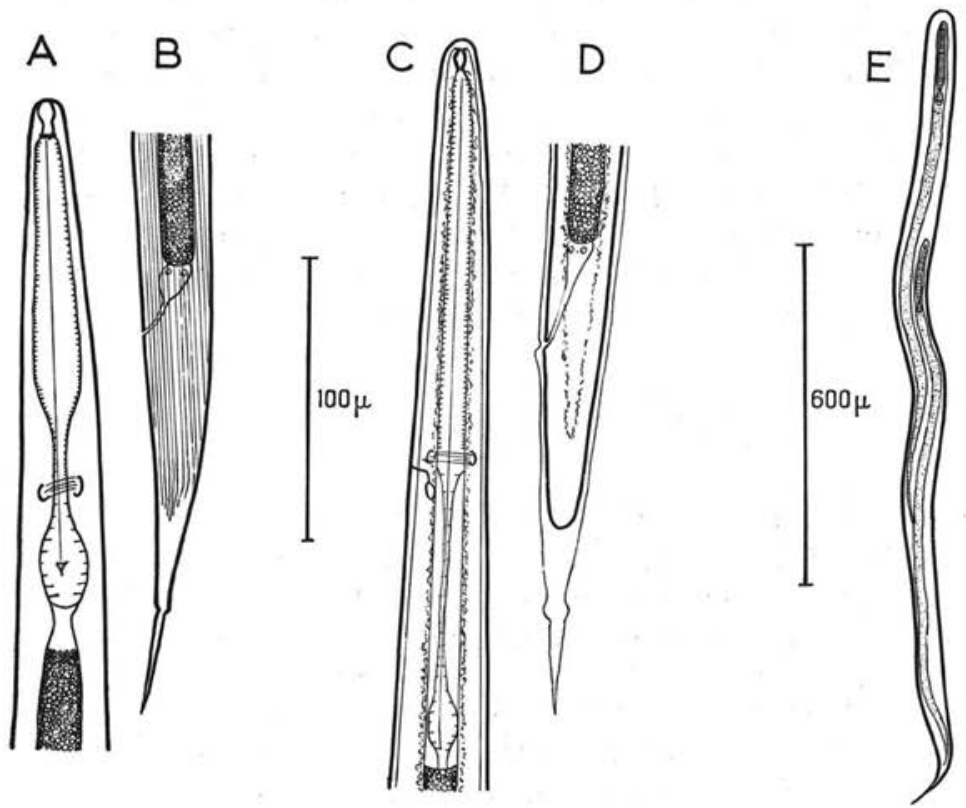

Fig. 5. - Rhabdias gemellipara. Larves hétérogoniques. A et B : Extrémités antérieure et postérieure d'une large rhabditoïde extraite artificiellement de la dépouille maternelle (4 ${ }^{\mathrm{e}}$ jour). $\mathrm{C}$ et D : Extrémités antérieure et postérieure d'une larve infestante, incluse dans la dépouille du stade précédent ( $5^{\bullet}$ jour $)$. -E : Dépouille maternelle contenant les deux larves sœurs en cours d'évolution ( $4^{\circ}$ jour).

lique et se terminent à $75 \mu$ de la pointe caudale. Ces stries coupent les stries transversales normales, si bien que la cuticule semble couverte de petits reliefs régulièrement carrés. Le corps est long de $955 \mu$ et large de $30 \mu$. L'œsophage, long de $180 \mu$, est formé d'un pharynx, d'un corpus, d'un isthme et d'un bulbe longs respectivement de $12 \mu, 100 \mu, 40 \mu$ et $28 \mu$. Anneau nerveux et pore excréteur respectivement à 195 et $160 \mu$ de l'apex. Ebauche génitale, longue de 
$50 \mu$, avec partie moyenne située à $545 \mu$ de l'extrémité antérieure. Queue longue de $138 \mu$, brusquement rétrécie en pointe fine à $40 \mu$ de son extrémité.

\section{$7^{\circ}$ Larve infestante.}

Aussitôt après la mue qui a lieu le $5^{\circ}$ jour, la larve entre en diapause et sa morphologie devient très stable. Elle reste incluse dans la cuticule striée du stade précédent, mais a, elle-même, une cuticule normale. Elle est un peu plus fine et un peu moins longue, mais les seules modifications importantes portent sur la région œsophagienne et sur la queue. Le corpus, l'isthme et le bulbe œsophagiens fusionnent pour donner un œsophage cylindrique long $(260 \mu)$ et assez grêle. La portion pharyngée donne naissance à une très petite capsule buccale en forme de calice. L'anneau nerveux et le pore excréteur se rapprochent l'un de l'autre (anneau nerveux à $95 \mu$ et pore excréteur à $102 \mu$ de l'apex). La queue est beaucoup plus courte $(80 \mu)$ et se termine par une pointe presque arrondie.

\section{Discussion}

La systématique des Rhabdias semble actuellement à peu près inextricable. Environ 37 espèces différentes ont été décrites, mais beaucoup ne sont connues que par la femelle parasite. Celle-ci a une morphologie très homogène, quelle que soit l'espèce, et a cependant des variations individuelles très importantes suivant son état de maturité. Il est donc probable qu'un bon nombre d'espèces sont synonymes. Inversement, il semble a priori peu vraisemblable, pour des Nématodes d'Amphibiens ou de Reptiles, qu'une espèce anglaise, telle que $R h$. sphaerocephala Goodey $1924 b$, puisse se trouver à Costa-Rica (Brenes et Bravo Hollis 1959), ou qu'une espèce américaine $R$ h. ranae Walton 1929 puisse se trouver au Pakistan (Gupta $1960)$.

Nous croyons cependant que nos deux espèces sont nouvelles, d'une part parce que nous ne connaissons pas encore d'exemple d'un Nématode d'Amphibien ou de Reptile malgaches qui ne soit pas endémique et, d'autre part et surtout, parce que la morphologie semble, dans les deux cas, un peu particulière :

$1^{\circ}$ Chez la première espèce, parasite de Rana, l'œsophage est constitué de deux parties très distinctes, une antérieure courte et étroite, une postérieure, plus longue et plus large. Il semble n'y avoir que 4 espèces ayant une morphologie comparable : a) Rh. sphaero- 
cephala Goodey 1924 b, parasite de Bufo en Angleterre ; mais l'extrémité céphalique présente une dilatation particulière, les glandes excrétrices sont peu visibles et, accessoirement, les dimensions sont très différentes; b) $R h$. hylae Johnston et Simpson 1942, parasite de Hyla et de Limnodynastes en Australie, mais la cuticule est ornée de stries longitudinales et l'espèce est décrite comme ayant huit structures dentiformes en avant de l'œsophage ; c et d) Rh. entomelas (Duj. 1845) et Rh. dujardini (Maupas 1916), tous deux parasites d'Anguis en Europe, se distinguent immédiatement par la capsule buccale bien développée et par le cycle évolutif bien différent.

Nous proposons donc le nom de Rhabdias madagascariensis n. sp. pour notre espèce.

$2^{\circ}$ En ce qui concerne la seconde espèce, parasite de Caméléon, le caractère donné par la division de l'œsophage est associé à d'autres particularités morphologiques (capsule buccale de la femelle parasite, stries cuticulaires longitudinales de la larve issue des femelles libres) et biologiques (endotokie et gémelliparité).

La diagnose peut donc être limitée à $R h$. chamaeleonis (Skrjabin 1916), décrit d'Afrique, qui est beaucoup plus grand $(24 \mathrm{~mm}$.) et a de grosses ailes latérales; la queue est fortement déportée dorsalement.

Mais c'est surtout Rh. entomelas (Duj. 1845) qui, bien que la femelle parasite ait une capsule buccale beaucoup plus développée, présente des affinités tout à fait remarquables avec notre espèce. Le cycle évolutif, suivi par Linstow (1885) et confirmé par Maupas (in Seurat 1916, p. 341, et 1920, p. 123-124), comporte des femelles libres qui ne produisent que deux œufs fécondés. Les deux larves se nourrissent aux dépens des tissus de la mère et semblent seulement se dégager plus rapidement de la dépouille cuticulaire de celleci. Les larves infestantes restent incluses dans la cuticule du stade précédent, qui porte de fortes stries longitudinales. L'évolution, y compris ses particularités les plus curieuses, est donc exactement superposable à celle que nous avons observée.

Il serait tentant de reprendre le genre Entomelas proposé par Travassos (1930), car la biologie de ces espèces est tout à fait distincte de celle des autres Rhabdias, mais de nombreux Rhabdias sensu stricto, principalement parmi les formes asiatiques, ont une capsule buccale plus grande que celle de notre espèce, et il ne reste guère de critères morphologiques pour caractériser le genre. Nous croyons donc, au moins à titre provisoire, devoir renoncer à l'emploi du genre Entomelas et désigner notre espèce sous le nom de Rhabdias gemellipara $\mathrm{n}$. sp. 


\section{RÉSUMÉ}

Description de Rhabdias madagascariensis n. sp., parasite de Rana mascareniensis. Comme il est fréquent chez les formes parasites d'Amphibiens, l'espèce a un cycle hétérogonique. L'œsophage de la femelle parasite, constitué de deux portions très distinctes, permet de limiter la diagnose à un petit nombre d'espèces.

Description de Rhabdias gemellipara n. sp., parasite de Chamaeleo parsonii à Périnet. Le cycle comporte plusieurs éléments curieux : la femelle libre ne donne naissance qu'à deux larves. Celles-ci mûrissent en dévorant les tissus de la mère. La larve infestante entre en diapause, associée avec la larve sœur, à l'intérieur de la cuticule maternelle, et chacune d'elles est protégée en outre par la dépouille cuticulaire fortement striée du stade précédent. L'hôte définitif s'infeste par voie buccale.

Il est curieux de constater qu'une espèce d'origine tout à fait différente, $R$. entomelas, parasite de l'Orvet en France, présente les mêmes particularités biologiques. Les analogies morphologiques entre les deux espèces semblent cependant trop faibles pour qu'il soit possible de reprendre le genre Entomelas.

\section{BiBLIOGRAPHIE}

Brenes (R. R.) et Bravo Hollis (M.), 1959. - Helmintos de la Republica de Costa Rica, VIII Nematoda 2. Algunos nematodes de Bufo marinus marinus (L) y algunas consideraciones sobre los generos Oxysomatium y Aplectana. Rev. Biol. Trop., VII, 35-55, fig. 1-12.

Chabaud (A. G.) et Petrer (A. J.), 1961. - Evolution et valeur systématique des papilles cloacales chez les Nématodes Phasmidiens, parasites de Vertébrés. C.R. Acad. Sc., CCLII, 1684-1686.

Goopey (T.), 1924, a. - The anatomy and life-history of the Nematode Rhabdias fuscovenosa (Railliet) from the Grass Snake Tropidonotus nutrix. J. Helminth., II, 51-64, fig. 1-8.

- 1924, b. - Two new species of the Nematode genus Rhabdias. J. Helminth., II, 203-208, fig. 1-11.

GuPTA (S. P.), 1960. - Nematode parasites of Vertebrates of East Pakistan, VI. Amplicaecum cacopi, Thelandros sp., Rhabdias ranae, and Oswaldocruzia melanosticti sp. nov., from Frogs. Can. J. Zool., XXXVIII, 745750 , fig. 1-14.

Johnston (T. H.) et Simpson (E. R.), 1942. - Some Nematodes from australian Frogs. Trans. Roy. Soc. South Aust., LXVI, 172-179, fig. 1-16.

Linstow (O. von), 1885. - Zur Kenntniss des Genus Angiostomum. Arch. Naturg., Berlin, LI, v. 1, 235-255, pl. 13-15, fig. 1-31. 
Seurat (L. G.), 1916. - Contribution à l'étude des formes larvaires des Nématodes parasites hétéroxènes. Bull. Sc. France et Belg., XLIX, 297-377, fig. 1-14.

- 1920. - Histoire Naturelle des Nématodes de la Berbérie, Stamel, Alger, $221+$ vi pp., fig. 1-34.

Skrjabin (K. I.), 1916. - Parasitic Trematodes and Nematodes collected by the expedition of Prof. V. Dogiel and I. Sokolov in British East Africa. Nauch Rezul't Zool. Eksped. (V. A. Dogiel i I, I. Sokolov), Brit. Vost. Afriku i Ugandu, 1914, art. 4, 157 pp., fig. A-C, pl. 1-10, fig. 1-82. Texte anglais, p. 99-157.

Travassos (L.), 1930. - Perquizas helminthologicas realisadas em Hamburgo. VII Notas sobre os Rhabdiasoidea Railliet, 1916. Mem. Inst. Osw. Cruz, XXIV, 161-181 + pl. 51-57.

Muséum National d'Histoire Naturelle. Laboratoire de Zoologie des Vers et Institut Pasteur de Madagascar 